\title{
Affective transfer as a function of reward and sex of subject
}

\author{
MELVIN H. MARX and KATHLEEN MARX \\ University of Missouri, Columbia, Missouri 65201
}

\begin{abstract}
A total of 130 high school psychology students first rated, with knowledge of results, 16 facial photographs for extraversion and observed their partners similarly rate a like number of faces. In a transfer test all subjects then rated the same 32 faces for "likability," "intelligence," or "character." Students, particularly female, showed a greater tendency to give elevated ratings to faces on which their extraversion judgment had been correct (rewarded). The type of transfer test did not make a difference. The results are interpreted as supporting the general proposition that reward can have affective (noncognitive) transfer effects and as raising some interesting questions concerning sex differences.
\end{abstract}

In a recent study (Marx, 1978), high school students were first asked to rate a number of facial photographs on each of four personality traits. Frequency norms, indicating the degree to which each trait had earlier been assigned to the faces by college students, were provided following the ratings on a given face. Students worked in pairs. Half of the subjects made the judgments on the first set of faces, with their partners observing and scoring as correct any response that was close to the norm; these roles were reversed for the second set of faces. All subjects, working individually, were then shown the same 32 faces again and asked to select the "most outstanding trait," of the four previously rated, for each face. When a single previously correct trait was compared with the trait that had been assigned the highest frequency score for that same face, male performers predominantly selected previously correct traits. Male observers, and both female performers and female observers, predominantly selected high-frequency traits. These results were interpreted as offering some support for the proposition that reward can have behavioral effects independent of the information that it necessarily provides; in other words, that it has an affective as well as cognitive component.

The present experiment was designed to extend the

We thank William Sympson, psychology teacher at Marathon, Florida, High School, and Joan Girnis and Gary Blodick, psychology teachers at Naples, Florida, High School, for their cooperation in arranging student participation in this experiment as a part of their course work. Familiarization of the students with research procedures and interpretations was further facilitated by the subsequent explanation, by the first author, of the general problem and procedure and the presentation of some preliminary results. This was done either in person or by means of a report given to the teacher. This research was supported in part by Research Career Award 5-K-6-MH-22023 from the National Institute of Mental Health and a grant from the U.S. Army Research Institute for the Behavioral and Social Sciences to the first author. The opinions expressed herein are those of the authors and in no way are to be considered as endorsed by the U.S. Army. investigation of transfer as a function of reward. This transfer is here called "affective," to distinguish it from the more commonly investigated cognitive variety, on the assumption that it is mediated by some kind of positive emotional reaction produced by reward and associated with concurrent S-R relationships.

Judgment of personality traits from facial photographs was used as the vehicle for analysis of the hypothesized reward-transfer function. Performers and observers were again paired in training, and each of 32 facial photographs was rated for extraversion. Immediate knowledge of results was provided in the form of "correct" ratings. The influence of reward was assumed to be strongest for performers, whose responses were most directly affected. On the transfer test, all subjects were asked to rate the same faces on one of three personality variables ("likability," "intelligence," and "character"). These traits were intended to vary with respect to increasing difficulty of rating, as listed above, on the assumption that the relatively more explicit traits, likability and intelligence, would be easier to rate than the deliberately vague "character." It was anticipated that amount of transfer, in the form of elevated test ratings, would be correlated with degree of difficulty of rating, on the ground that the more difficult ones would be more open to influence from prior reward, and that, on the basis of the earlier results (Marx, 1978), more transfer would occur for male subjects.

\section{METHOD}

\section{Subjects}

A total of 130 high school students (57 male, 73 female) in six psychology classes served as subjects. They were trained and tested in their normal classroom setting. Class size ranged from 15 to 28 .

Design

Groups were defined on the basis of the trait involved in the transfer test. Two classes $(n=36)$ were given the "likability" 
test, two others $(n=50)$ the "intelligence" test, and the last two $(n=44)$ the "character" test. The classes were otherwise treated the same.

\section{Task and Procedure}

The subject's task in both parts of the experiment was to make a personality judgment from a facial photograph presented by a slide projector. All classes were first asked to rate the degree of extraversion (low, moderate, or high) evident in a series of 32 facial photographs of college-age and more mature persons. Preliminary research with college students who had judged the same facial photographs provided the basis for rating each face as low, moderate, or high in extraversion; these "correct" ratings were given to the subjects orally after all had responded to the face shown.

Students used their identification numbers or initials on their record sheets in order to minimize personal identification.

For the training phase, subjects were placed in like-sexed pairs (with one exception) so that they could take turns "performing" (making the extraversion judgment) and "observing" (watching their partners write down the judgment and then circling the answer if it was the same as the experimenter called out). The person on the left of each pair served as the performer for the first 16 faces, the person on the right as the performer for the second set of 16 faces. Each set contained eight male and eight female faces and approximately the same number of low, moderate, and high ratings, randomly ordered.

Each face was presented for approximately $10 \mathrm{sec}$. Extraversion was simply defined as "the tendency to be socially outgoing, to relate easily to other people." Observers as well as performers were urged to study each face and note the outcome of the judgment "because the observer needs to score each of the judgments made by the performer but also because we want to see whether your judgments improve as a result of $r$. tice with making and observing them." This mild deception, which was deemed necessary if the intent of the experiment was to be carried out, was subsequently explained to the classes, along with a discussion of the difficulty of actually making personality judgments from photographs.

In the second (transfer) phase, the students worked individually and no knowledge of results was provided. Subjects were simply told that we were interested in determining the degree of likability, intelligence, or character that was evident in each of the faces. Ratings were on a 10-point scale, from 0 to 9 , and subjects were encouraged to spread their ratings over the entire scale.

The two parts of the experiment were presented as separate experiments in order to avoid biasing the test results by subjects' expectancies concerning the relationship between them.

\section{RESULTS}

Tests of proportions of subjects falling into one category or another were used. This procedure was adopted because of the widely varying contributions of the subjects to the various categories, so that the more rigorous assumptions of ANOVA, such as of heterogeneity of variance, would be questionably satisfied.

Overall, distribution of right and wrong extraversion judgments was surprisingly close to 50-50, in spite of the $1 / 3$ chance a subject had of being right on any given response, on a purely statistical basis. The total of 130 subjects in fact averaged 7.48 correct judgments on the 16 faces $(46.7 \%)$. This fact probably reflects some bias in making such judgments that is common to the high school students sampled and the college students on whom the extraversion-judgment norms were based.
In spite of this even distribution of right and wrong judgments, there were numerous cases of disproportionate distribution. For example, there were two instances in which all eight extraversion judgments for one type of face (male or female) had been wrong and seven instances in which only one judgment had been correct; thus this variable (sex of the face shown) was collapsed, in order to build up the number of instances analyzed for each subject and so permit more reliable categorization.

Of the many possible comparisons of categories based on transfer-test scores, only the two that seem to be the most representative will be presented. In the simpler single-subject measure, there was first a comparison for each subject of the test scores made on previously correct items with those made on his or her own previously incorrect items, yielding a mean right-wrong score. This mean score was then compared with the corresponding score based on his or her observation data, considered as a control. If the performer difference was greater than the observer difference, the subject was categorized as a positive instance (i.e., in accordance with prediction); if not, as a negative instance. The test for statistical reliability of differences between proportions described by Bruning and Kintz (1977, p. 120ff) was then applied. For this purpose a 50-50 chance baseline was used. Tied scores were not counted in either this or the other analysis.

The second, more complex, paired-subject measure controlled for the particular face being judged by comparing each performer's correct-response scores with his or her partner's (observer) scores. If the performer mean was greater, the subject was categorized as positive; if not, as negative. A second test was then applied to the same two (paired) subjects by using the corresponding scores for the referent subject's wrong scores. In order to make the categorizing as sensitive as possible, the subject was categorized as positive, on this second test, if the relative difference between his or her wrong and the observing partner's score was less than the right-score difference already obtained; otherwise, the subject was categorized as negative on this second test. Statistical evaluation was then applied using a test for differences between proportions when the same subjects are given two tests (Edwards, 1960, p. $57 \mathrm{ff})$.

\section{Test Structure}

Single-subject measure. The proportion of positive to negative instances for likability, intelligence, and character groups, respectively, was: $20: 14$ (59\% positive), $30: 20$ (60\% positive), and 29:15 (66\% positive). Thus all groups showed a tendency in the predicated direction, although only the likability $(p<.01)$ and character $(p<.02)$ differences were clearly reliable.

Paired-subjects measure. The three groups showed these proportions of positive-negative to negativepositive patterns of proportions (the two key propor- 
tions used in the test): likability, 11:6 (65\% positivenegative); intelligence, $22: 8$ (73\% positive-negative); and character, $22: 13$ (63\% positive-negative). By this analysis only the intelligence group showed a clearly reliable difference $(p<.01)$, although, again, all of the differences were in the predicted direction.

\section{Sex of Subject}

Single-subject measure. Male subjects showed a $31: 24$ (57\% positive) and female subjects a 48:25 (66\% positive) ratio of positive (predicted) to negative instances. Only the female difference was reliable $(\mathrm{p}<.01)$.

Paired-subjects measure. Positive-negative and negativepositive proportions were $21: 14$ (60\% positive-negative) for males and $34: 13$ ( $72 \%$ positive-negative) for females. Again, only the female subjects showed a reliable difference $(p<.01)$.

\section{Overall Comparison}

Single-subject measure. The ratio of positive to negative instances was 79:49 (62\% positive), reliable at $\mathrm{p}<.01$

Paired-subjects measure. The proportion of positivenegative to negative-positive instances was 55:27 (67\% positive-negative), reliable at $\mathrm{p}<.01$.

\section{DISCUSSION}

Three conclusions are immediately indicated by the pattern of results: (1) There is no indication that the test-structure variable was at all effective in differentiating the groups, but all three test conditions seemed to produce the predicted results. (2) Female subjects were more likely to give elevated test ratings following reward in training than male subjects, although males did show the same tendency. (3) Overall, there was clearly a strong tendency in the predicted direction, thus providing further support for the proposition that reward can have affective (noncognitive) consequences that are not generally recognized by human learning researchers and theorists.

Why females in this study should clearly show strong transfer effects whereas only males did in the previous study (Marx, 1978) is not readily evident. However, the apparent contradiction in results may be a function of procedural differences. In the earlier experiment, the particular kind of response that had been rewarded (a judgment of a given personality trait) was transferred more or less directly (i.e., the same trait name selected as the "outstanding trait" for that face), whereas in the present experiment, the transfer was to a more distinctly different response dimension. It remains for future research to analyze this difference, and to explore the intriguing possibility that males are more enamored of their own (rewarded) responses and females are more likely to be generous with their ratings following reward.

\section{REFERENCES}

Bruning, J. L., \& KINTZ, B. L. Computational handbook of statistics (2nd. ed.). Glenview, Ill: Scott, Foresman, 1977.

EDWARDs, A. L. Experimental design in psychological research (2nd. ed.). New York: Holt, Rinehart, \& Winston, 1960.

MARX, M. H. Transfer of rewarded responses in personality judgments. Bulletin of the Psychonomic Society, 1978, 2, 112-114.

(Received for publication May 9, 1978.) 